\title{
Extraordinary Transmission and Enhanced Emission with Metallic Gratings Having Converging-Diverging Channels
}

\author{
Arvind Battula, ${ }^{1}$ Yalin Lu, ${ }^{2}$ R. J. Knize, ${ }^{2}$ Kitt Reinhardt, ${ }^{3}$ and Shaochen Chen ${ }^{1}$ \\ ${ }^{1}$ Department of Mechanical Engineering, Center for Nano and Molecular Science and Technology, \\ University of Texas, Austin, TX 78712, USA \\ ${ }^{2}$ Physics Department, Laser Optics Research Center, USAF Academy, CO 80840, USA \\ ${ }^{3}$ Air Force Research Laboratory, AFOSR/NE, 875 North Randolph Street, Suite 326, Arlington, VA 22203, USA
}

Received 22 October 2007; Accepted 26 November 2007

Recommended by Weili Zhang

Transmission metallic gratings having the shape of converging-diverging channel (CDC) give an extra degree of freedom to exhibit enhanced transmission resonances. By varying the gap size at the throat of CDC, the spectral locations of the transmission resonance bands can be shifted close to each other and have high transmittance in a very narrow energy band. Hence, the CDC shape metallic gratings can lead to almost perfect transmittance for any desired wavelength by carefully optimizing the metallic material, gap at the throat of CDC, and grating parameters. In addition, a cavity surrounded by the CDC shaped metallic grating and a one-dimensional (1D) photonic crystal (PhC) can lead to an enhanced emission with properties similar to a laser. The large coherence length of the emission is achieved by exploiting the coherence properties of the surface waves on the gratings and PhC. The new multilayer structure can attain the spectral and directional control of emission with only p-polarization. The resonance condition inside the cavity is extremely sensitive to the wavelength, which would then lead to high emission in a very narrow wavelength band. Such simple 1D multilayer structure should be easy to fabricate and have applications in photonic circuits, thermophotovoltaics, and potentially in energy efficient incandescent sources.

Copyright (c) 2007 Arvind Battula et al. This is an open access article distributed under the Creative Commons Attribution License, which permits unrestricted use, distribution, and reproduction in any medium, provided the original work is properly cited.

In late 1980s, the concept of photonic crystals emerged, which initiated new studies of gratings, as examples of onedimensional (1D) periodic media $[1,2]$. Since then, some theoretical studies have been done on the transmission gratings, but they did not receive much attention because it was thought that the transmission through the subwavelength apertures is very low according to the standard aperture theory by Bethe [3], which states that the transmission through a subwavelength circular hole $(r \ll \lambda)$ in an infinitely thin perfectly conducting metal sheet would scale uniformly with the ratio of $r$ to $\lambda$ to the power of four. But, an extraordinary transmission (EOT) of several orders of magnitude more than Bethe's prediction has been reported through an array of subwavelength holes milled in an opaque metal screen [4]. The underestimation of transmission by Bethe's theory is because it is too idealized to consider the surface modes that might be involved and also propagating of evanescent modes that could be excited inside the holes [5]. Subsequently, enhanced transmission through hole arrays in metal films has been studied in great detail both theoretically and experimentally. This has sparked renewed interests in studying the transmission gratings (or slits), which are $1 \mathrm{D}$ version of the hole structures studied by [4], to explain the underlying physics for enhanced transmission. However, the transmission properties between slit and hole arrays have a fundamental difference. In a slit waveguide, there is always a propagating mode inside the channel, whereas in a hole waveguide all modes are evanescent when hole diameters are smaller than half the wavelength, and there is a cutoff frequency for transmission [6]. Hence, the slit and hole array structures have very different propagation mechanisms. But, it is believed in general that the EOT phenomenon is mainly due to the surface plasmons polariton (SPP) modes trapped at the interface of metal and dielectric. The SPPs are actually quanta of collective plasma oscillations localized at interface of a metal and dielectric [7]. However, not all agree on the same SPP mechanism for the enhanced transmission phenomena $[8,9]$. Nevertheless, according to several other theoretical and experimental studies, the enhanced transmission process through subwavelength metallic hole array (MHA) can be divided into three steps: the coupling of light to SPPs on the incident surface, transmission through the holes to the 
second surface, and then reemission from the second surface [5]. In addition, diffraction plays a central role in the transmission process through subwavelength holes or gratings. When a plane wave is incident on grating array, the diffraction leads to evanescent wave. After the transmitted evanescent wave reaches the far end of the array, it gets diffracted again producing a propagating transmitted wave. Therefore, the transmission enhancement occurs when the diffraction aids in coupling the incident light and the SPP modes of the metal structure [10]. It was also reported that for lamellar transmission metallic gratings, there are two transmission resonances: coupled SPP modes on both the horizontal surfaces of the metallic grating for $\lambda \sim d$, and cavity or waveguide modes located inside the slits for $\lambda \gg d[11]$. SPPs will be resonantly excited on the gratings only when the higher-diffraction order along with the incident parallel momentum equals SPPs momentum according to the equation $K_{\mathrm{SPP}}=K_{o} \sin (\theta) \pm m(2 \pi / d)$.

In thermal source, the light generation at the microscopic level is a spontaneous emission of photon when an emitter thermally excited relaxes to a lower state. Unlike the laser which produces highly directional and monochromatic light, thermal light source is isotropic with a broad spectrum. But the thermal source of light is coherent in the near field, that is, within a distance from the surface that is much smaller than the emission peak wavelength of the spectrum [12-14]. The coherence is due to the role of surface waves. Hence, a roughness or a grating on the surface can couple these waves to propagating waves, which will extend the coherence properties into the far field. Thus, by modifying the characteristics of the surface profile, the near field coherence properties can be extended to far field in a particular direction at a given wavelength. This has been observed first on a doped silicon grating supporting a surface plasmon polariton (SPP) [15]. Similarly, a peak in the thermal emission by gratings on ZnSe [16], gold [17], and SiC [18] was also observed. For these grating structures, it was noted that the excited surface waves could couple to the emitted radiation for the $p$ polarization only. No lobe of emission has been observed for $s$-polarization since, in this case, the SPPs or surface waves cannot be present [19].

In the present study, we propose a subwavelength grating with converging-diverging channel (CDC) for extraordinary transmission and in combination with one-dimensional (1D) $\mathrm{PhC}$ for enhanced emission. For the emission, there is a cavity between the CDC grating and the $1 \mathrm{D} \mathrm{PhC}$. Here, we could make use of the surface waves that could be present on both the metallic gratings and in the $\mathrm{PhC}$ structure. The 1D $\mathrm{PhC}$ considered is made of lossless dielectrics $\left(\mathrm{SiO}_{2}\right.$ and InSb) with refractive indices given by [20] $n_{\mathrm{SiO}_{2}}=1.46$ and $n_{\text {InSb }}=3.95$. A freely available MIT photonic band (MPB) package was used for calculating the photonic band structure (PBS) of the PhC. The first or lower bandgap in the PBS is between the normalized frequency range of 0.136 to 0.217 . So, a $\mathrm{PhC}$ having a unit cell thickness "a" of $100 \mathrm{~nm}$ has first bandgap wavelength range that is between $460 \mathrm{~nm}$ to $735 \mathrm{~nm}$.

According to Kirchhoff's law, the directional spectral emissivity $\left(\varepsilon_{\lambda, \theta}\right)$ can be determined by using $1-\rho_{\lambda, \theta}-\tau_{\lambda, \theta}$ [21], where the directional spectral reflectance, $\left(\rho_{\lambda, \theta}\right)$, and the

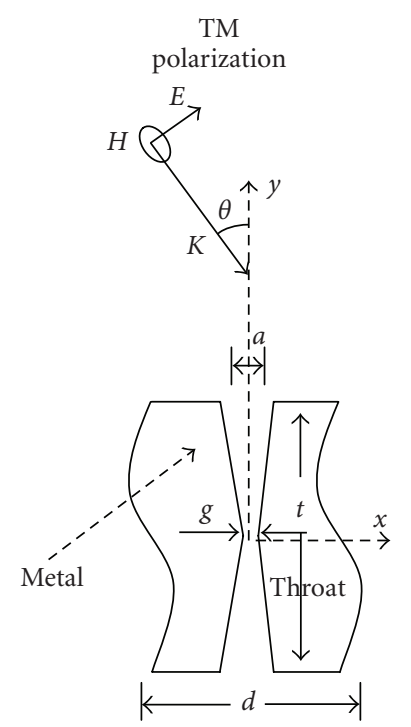

FIGURE 1: Schematic view of the lamellar transmission metallic grating with converging-diverging channels in vacuum with grating parameters as period $(d)$, aperture $(a)$, and thickness $(t)$.

directional spectral transmittance, $\left(\tau_{\lambda, \theta}\right)$, are evaluated by using a plane monochromatic wave incident from the air at an angle of incidence " $\theta$ " as shown in Figure 1. In the figure, we show a schematic view of a metallic grating with CDC in vacuum that was studied with the definition of different parameters: the period of grating $(d)$, the aperture width $(a)$, the grating thickness $(t)$, and the gap at the throat $(g)$. The gap sizes were varied from $0.5 \mu \mathrm{m}$ to $5 \mathrm{~nm}$. It is plausible to use Maxwell's equation when the absolute lower limit of the length of macroscopic domain is $10 \mathrm{~nm}$ [22]. Therefore, it is assumed that the results obtained in the present study for gap of $5 \mathrm{~nm}$ will be rational. Nevertheless, it should be pointed out that the effects discussed in this study do apply for any other range of grating parameters provided that the aperture width is very small in comparison to the grating period. Also, the frequency of incident light has to be well below the plasma frequency of the metal [11]. The dielectric function of metals described in this study is from the tables reported in $[20,23]$. The transmission for metallic lamellar gratings with transverse electric (TE) polarization suffers a cutoff wavelength [24]. Hence, we have analyzed the metallic gratings with only TM polarization (magnetic field vector parallel to the gratings) in order to study whether the enhanced transmission could be achieved for any desired wavelength with the CDC structure. The resulting governing equation for the time harmonic electromagnetic fields, with the dependent variable as magnetic field in the $z$-direction $\left(H_{z}\right)$, is same as Helmholtz equation. Commercially available finite element software (FEMLAB 3.1i) was used for solving the governing equation. The $2 \mathrm{D}$ computational domain is surrounded by either a periodic boundary with perfect electric condition or a perfectly matching layer (PML) [25]. Since the proposed study has a structure that is periodic in $1 \mathrm{D}$ and has the length of the grating larger than the period of the grating, then the resulting computational domain would be a $2 \mathrm{D}$ domain. The 


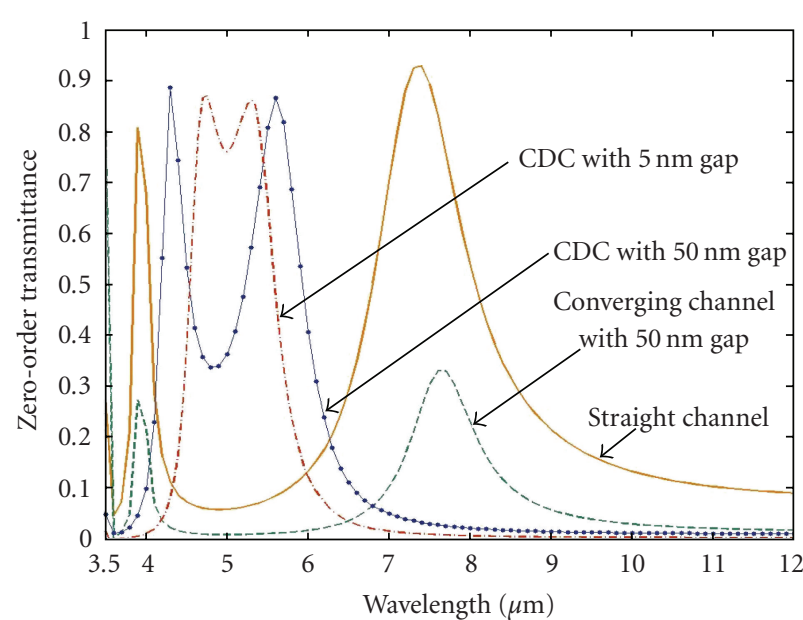

(a)

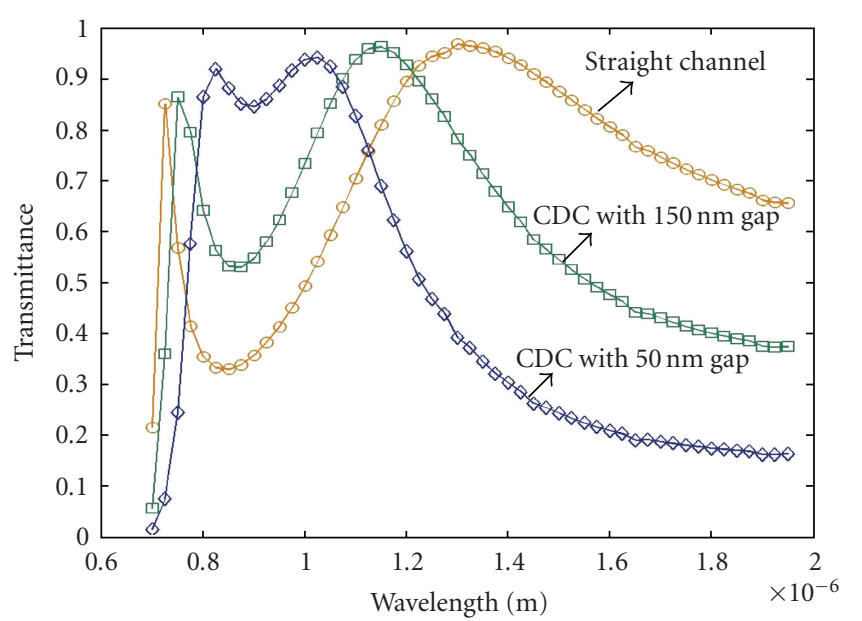

(b)

Figure 2: Zero-order transmittance for a normal incident plane wave on lamellar gratings in vacuum for different channel configurations, but with the same grating parameters as (a) $d=3.5 \mu \mathrm{m}, a$ $=0.5 \mu \mathrm{m}$, and $t=3.0 \mu \mathrm{m}$ in gold film; (b) $d=650 \mathrm{~nm}, a=300 \mathrm{~nm}$, and $t=500 \mathrm{~nm}$ in silver film.

transmittance $(\tau)$ and reflectance $(\eta)$ of the CDC grating were calculated from the obtained electromagnetic field distributions as follows:

$$
\tau=\frac{\left(H_{z} \cdot H_{z}^{*}\right)_{P 1, F}}{\left(H_{z} \cdot H_{z}^{*}\right)_{P 1, I}}, \quad \eta=\frac{\left(Q \cdot Q^{*}\right)_{P 2, F}}{\left(H_{z} \cdot H_{z}\right)_{P 1, I}},
$$

where $Q=\left(H_{z}-\left(\partial H_{z} / \partial y\right) /(i k \cos (\theta))\right), H_{z}$ is the magnetic field, $k$ is the wave vector, $\theta$ is the angle of incidence, and subscripts $F$ and $I$ indicate the incident and final transmitted energy. $P 1$ and $P 2$ indicate the planes below and above the grating.

Figure 2 shows the zero-order transmittance for normal incident radiation as a function of wavelength for different channel configurations. The CDC with $5 \mathrm{~nm}$ gap at the throat has a remarkable transmission in a very narrow wavelength band. The two transmission peaks for the straight channel are identified as the SPP and waveguide coupled resonance $(\sim 3.9 \mu \mathrm{m})$ and waveguide resonance $(\sim 7.4 \mu \mathrm{m})[11]$. Although the two transmission peaks seen for the converging channel are located at almost the same resonance wavelengths as for the straight channel, the transmittance is much lower, which is explained later. For CDC, the locations of transmittance peaks have changed in spectral positions and are approaching each other as the gap at throat decreases, as seen in Figure 2(a). This would lead to a high transmission in a very narrow wavelength band, and the transmittance is almost negligible everywhere else in the considered wavelength range. A closer look at Figure 2(a) would also tell that the resonances for CDC occur at wavelengths which had lower transmittance with a straight channel. Similar kind of behavior can be observed at different wavelengths for the silver gratings (Figure 2(b)).

By varying the angle of incidence " $\theta$," we can calculate the PBS and $\omega\left(k_{x}\right)$, of these transmission resonances or surface excitations of the metallic gratings. Using the photonic band structure (PBS), both the spectral position and the width of transmission peaks can be found. In Figure 3(a), we show the PBS of a CDC gold grating with $100 \mathrm{~nm}$ gap at the throat in vacuum. Also, we show the energetic positions of the SPP modes (white dashed lines) for a nearly flat metal surface. Figure 3(a) shows that there are two transmission resonance bands, with the higher-energy band following close to the SPP mode energetic positions and lower-energy band shifts in spectral location with the angle of incidence " $\theta$." For the transmission resonances, the phases across the grating have to be in-phase for a Fabry-Perot or waveguide kind of resonance to occur [26]. With further decrease in the gap at throat to $50 \mathrm{~nm}$ (see Figure 3(b)), the band at the lower energy has become more dependent on " $\theta$," and the transmittance above the first-order diffraction has increased more, but now over a shorter range of energy spectrum when compared to that of the $100 \mathrm{~nm}$ gap (see Figure 3(a)). When the gap at the throat of CDC reduces the high-transmission resonance, bands in the PBS change from their previous energy positions, and the bands appear to approach each other (see Figure 3 ). Hence, it should be pointed out that with very small gap at the throat, the transmission over the entire energy spectrum could be made high only in a very narrow band. The shifting of transmission resonance band with the gap at the throat of the CDC can be seen in Figure 4, where the transmittance of CDC aluminum grating as a function of wavelength in UV-visible range and gap size at the throat is shown. The full width at half maximum (FWHM) of the transmission peak shrinks with the reduction in the throat gap. In addition, the transmission peak magnitude declines with the throat gap. This is due to two things: (i) the shift of transmittance peak to lower wavelengths that is accompanied by (ii) the change in the channel shape, which might increase the skin depth that would subsequently increase the absorption. Also, it is to be noted that the slight reduction in the transmittance peaks happens in the UV-visible range, and it is not observed at higher wavelength range since the skin depth remains constant for those wavelengths.

The normalized magnitude of the $x$-component electric field $\left(\left|E_{x}\right|\right)$ along the center line of the channel at $\sim 6.35 \mu \mathrm{m}$ 


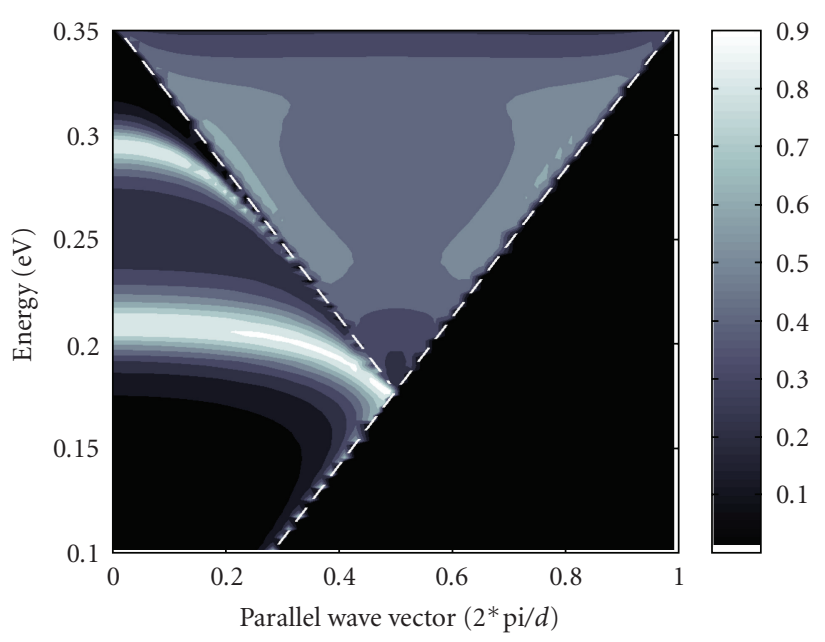

(a)

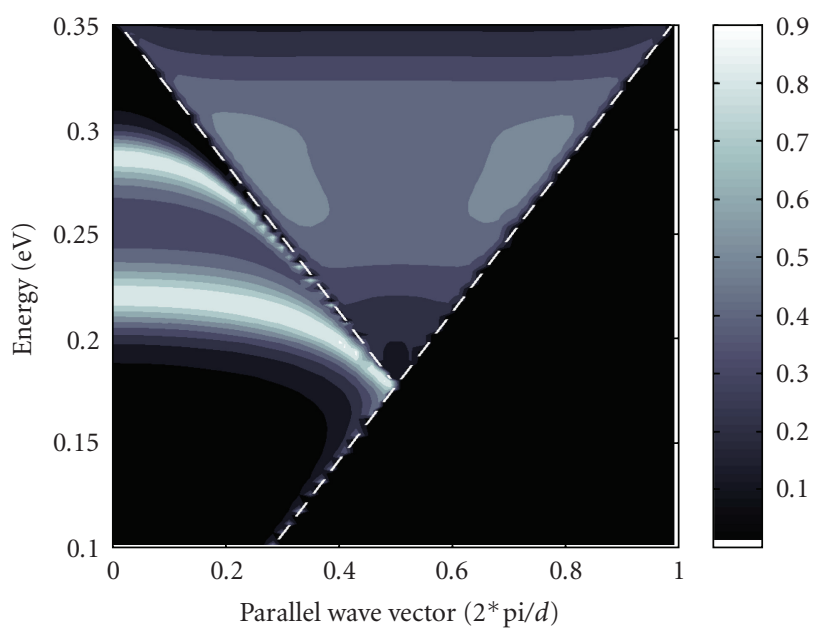

(b)

FIGURE 3: Photonic band structure of the surface plasmons responsible for the transmission resonances of gold gratings in vacuum with slit shape as (a) CDC with $g=100 \mathrm{~nm}$ and (b) CDC with $g=$ $50 \mathrm{~nm}$. The grating parameters are fixed at $d=3.5 \mu \mathrm{m}, a=0.5 \mu \mathrm{m}$, and $t=3.0 \mu \mathrm{m}$. Also, energetic positions seen in figures are (white dashed lines) of the SPP modes.

wavelength for the converging channel and CDC is shown in Figure 5. The variation of $\left|E_{x}\right|$ in the channel is proportional to the distribution of charges on the metallic grating channel surfaces. Also, by looking at the $\left|E_{x}\right|$ distribution along the channel would tell whether the wave inside the channel is in-phase or out-of-phase. In Figure 5(a), we show that when the gap is $500 \mathrm{~nm}$, the wave inside the channel is an out-ofphase standing wave. This is because the minimum magnitude (or node) is not at the center of the channel, and the magnitudes near the end of channels (or antinodes) are not equal. This indicates that at the end of channel, the dipoles have unequal strength which could be due to the destructive interference of the wave inside the channel [24]. The surface charges oscillate between these two antinodes leading to the

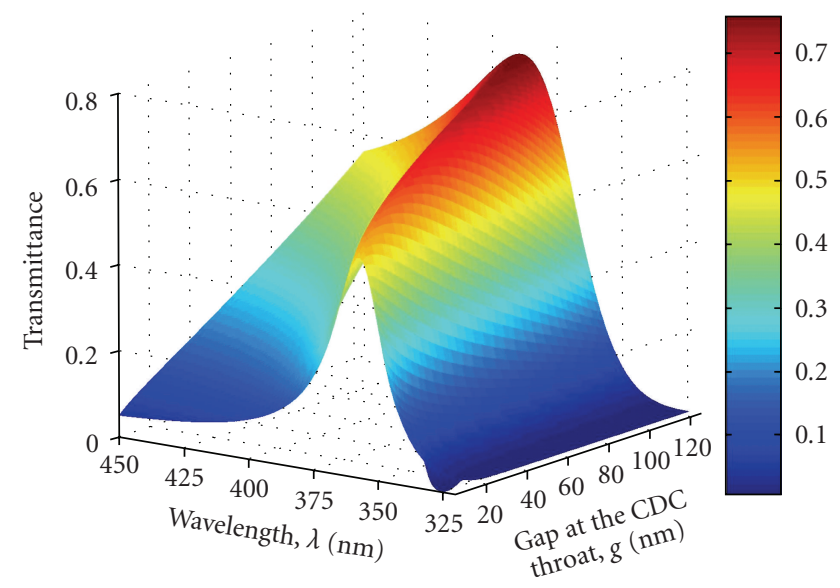

FIGURE 4: Transmittance in vacuum for CDC gratings as a function of the wavelength and distance at the gap of throat for the aluminum gratings having $d=315 \mathrm{~nm}, a=125 \mathrm{~nm}$, and $t=100 \mathrm{~nm}$.

surface current that couples to the incident light and transmits it to the other side of grating. With the decrease in gaps near the throat, the node moves further away from the center, and hence the wave inside the channel goes more outof-phase. Also, when the gap near the throat keeps decreasing, the dipole at the throat starts to gain in strength and becomes stronger than the dipole at the other end of channel. This causes very less net current or energy to flow out of the channel. Similarly, by looking at the $\left|E_{x}\right|$ distributions in Figure 5(b), we show that the wave inside the channel of CDC is a standing wave for different gaps near the throat. As the gap in CDC keeps decreasing, the node keeps moving toward the center of channel. Also, the magnitudes of $\left|E_{x}\right|$ at two antinodes are increasing and becoming equal. This shows that the CDC shape of the channel is aiding the wave inside the channel to become an in-phase standing wave. The condition for in-phase resonance inside the channel is given by [26] $2 K t+\Delta \phi=m 2 \pi$, where " $K$ " is the wave vector of the resonance, and " $\Delta \emptyset$ " is the phase change on the aperture of the slits. The role of throat is that for various gap sizes the length of the channel surface would change and hence would result in having different resonance wave vector. This can be seen from Figure 5(b) where it is shown that at $6.35 \mu \mathrm{m}$ wavelength the high transmittance happens at $200 \mathrm{~nm}$ gap near the throat, which has a standing wave inside the channel that is in-phase. A small decrease in the gap from $200 \mathrm{~nm}$ makes the standing wave inside the channel to go out-of-phase, and so the transmittance decreases. When the gap at throat becomes smaller, then the charges at throat gain strength, just like in the case of converging channel (see Figure 5(a)). This would not allow light to transmit more efficiently.

Normal emittance spectrum with $p$-polarization for the CDC grating with $5 \mathrm{~nm}$ gap and $1 \mathrm{D} \mathrm{PhC}$ with various cavity lengths can be seen in Figure 6(a). The $50 \mathrm{~nm}$ cavity length has one smaller emissive peak at lower wavelength. But, when the cavity length is increased to $100 \mathrm{~nm}$ then the emissive peak present at lower wavelength would increase in magnitude, and the spectral position of the peak moves 


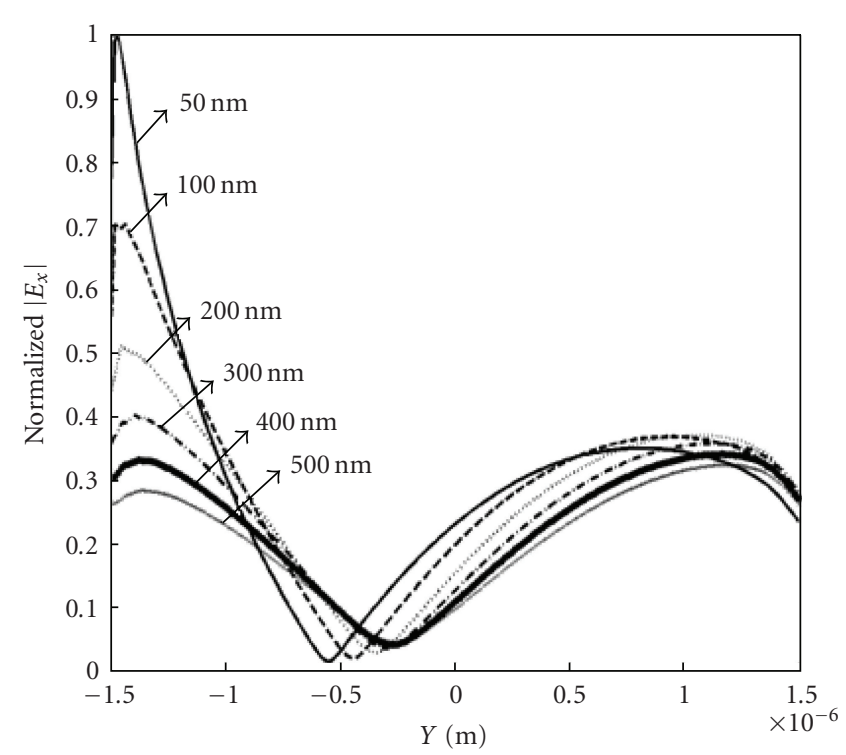

(a)

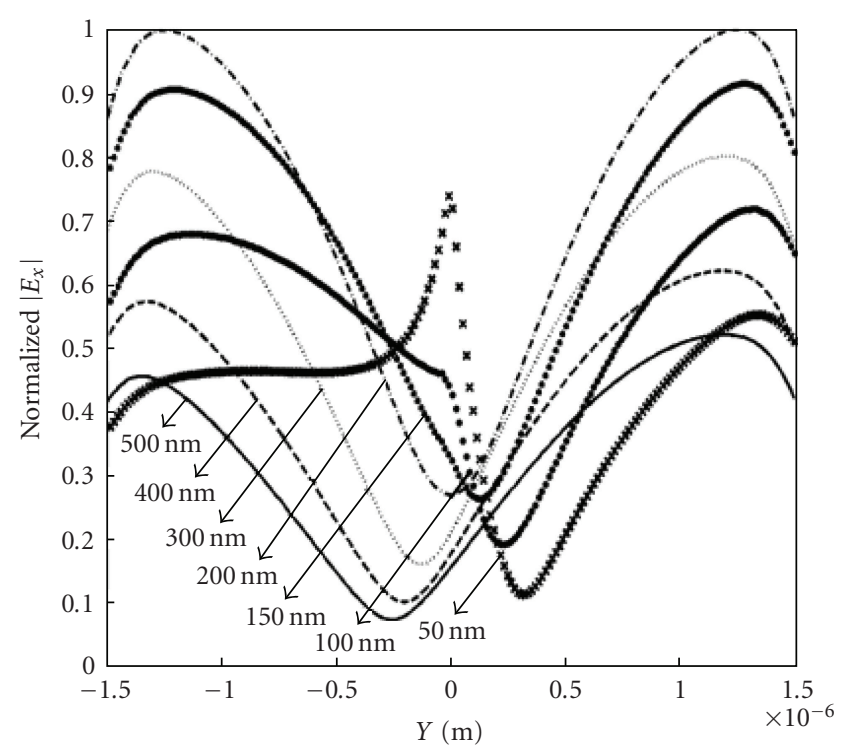

(b)

FIgURE 5: Normalized electric field $x$-component $\left(\left|E_{x}\right|\right)$ at an incident wavelength of $6.35 \mu \mathrm{m}$ along the channel center line of a gold grating in vacuum with (a) converging channel and (b) CDC for different gaps at the throat and grating parameters as $d=3.5 \mu \mathrm{m}, a$ $=0.5 \mu \mathrm{m}$, and $t=3.0 \mu \mathrm{m}$.

to a bigger wavelength or has a red shift. When the cavity length increases further to $200 \mathrm{~nm}$, then the only emissivity peak in the entire wavelength range of interest will have magnitude almost close to unity. The spectral position of the peak would have a further red shift. Figure 6(a) also shows that the $300 \mathrm{~nm}$ cavity length would have a highemissive peak with an additional red shift. The spectrum of normal emittance for the $320 \mathrm{~nm}$ cavity length has several

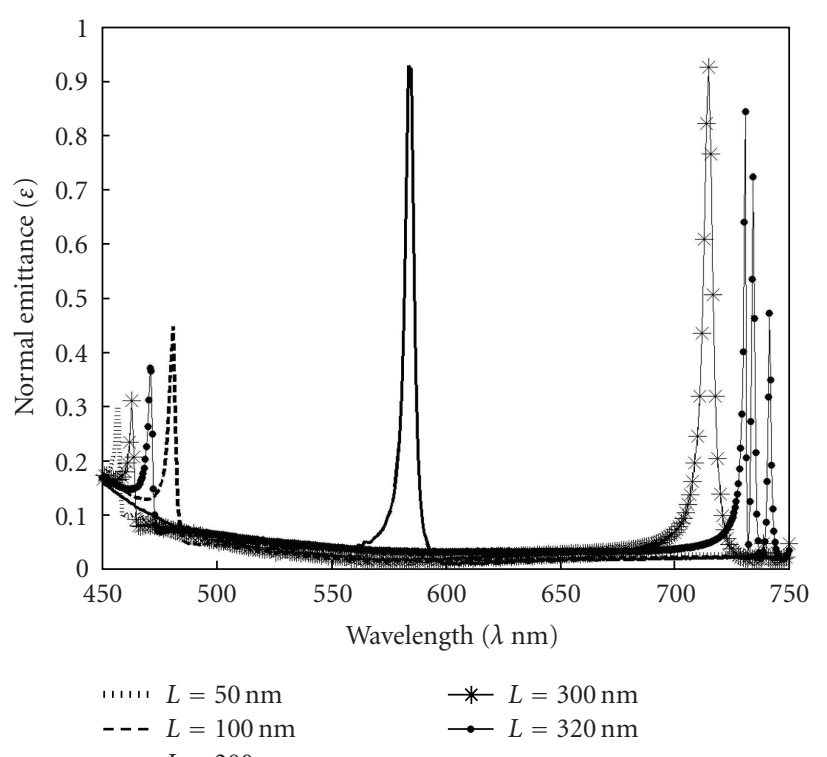

(a)

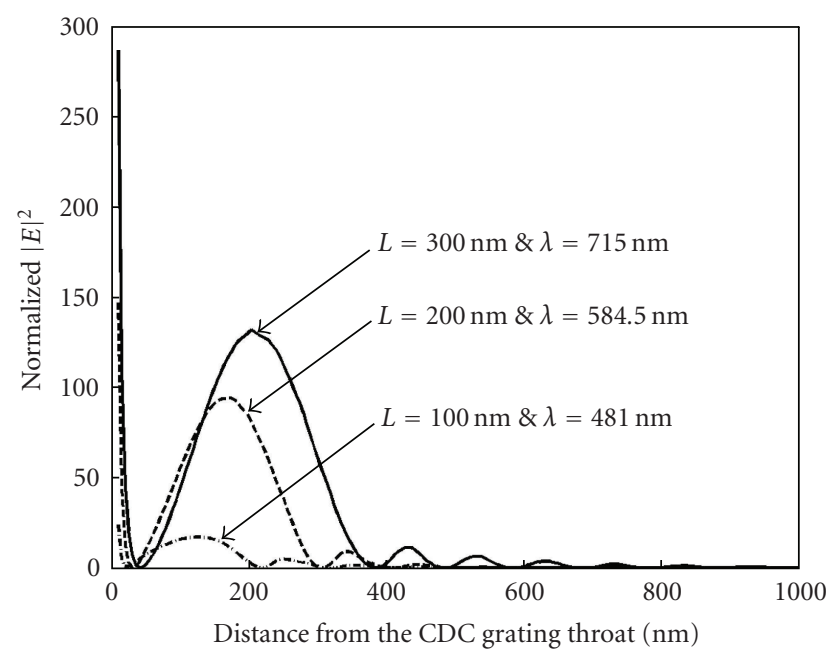

(b)

FIGURE 6: (a) Normal emittance spectrum with $p$-polarization in vacuum for Ag CDC grating having the parameters $\Lambda=250 \mathrm{~nm}$, $h=100 \mathrm{~nm}, t=100 \mathrm{~nm}, g=5 \mathrm{~nm}$, and a photonic crystal having the parameters $a=100 \mathrm{~nm}, d_{1}=d_{2}=50 \mathrm{~nm}$ with cavity length $L=$ $50 \mathrm{~nm}, L=100 \mathrm{~nm}, L=200 \mathrm{~nm}, L=300 \mathrm{~nm}, L=320 \mathrm{~nm}$; (b) electric field intensity distribution normalized to the incident along the line passing through the center of the CDC grating throat and into the photonic crystal with cavity lengths $L=100 \mathrm{~nm}, L=200 \mathrm{~nm}, L=$ $300 \mathrm{~nm}$, and at respective normal emissive peak wavelengths.

narrow peaks towards higher-band wavelength. The FWHM of the emissive peaks for the cavity length of $100 \mathrm{~nm}$ and $200 \mathrm{~nm}$ is the same and is around $4 \mathrm{~nm}$. For the $300 \mathrm{~nm}$ cavity length, the FWHM is around $5 \mathrm{~nm}$. Hence, Figure 6(a) shows that by varying the cavity length would result in the red shift of the emissive peak, and the FWHM of the peaks will be around 4-5 $\mathrm{nm}$. In addition, according to the WienerKhinchin theorem the width of the emission spectrum peak 


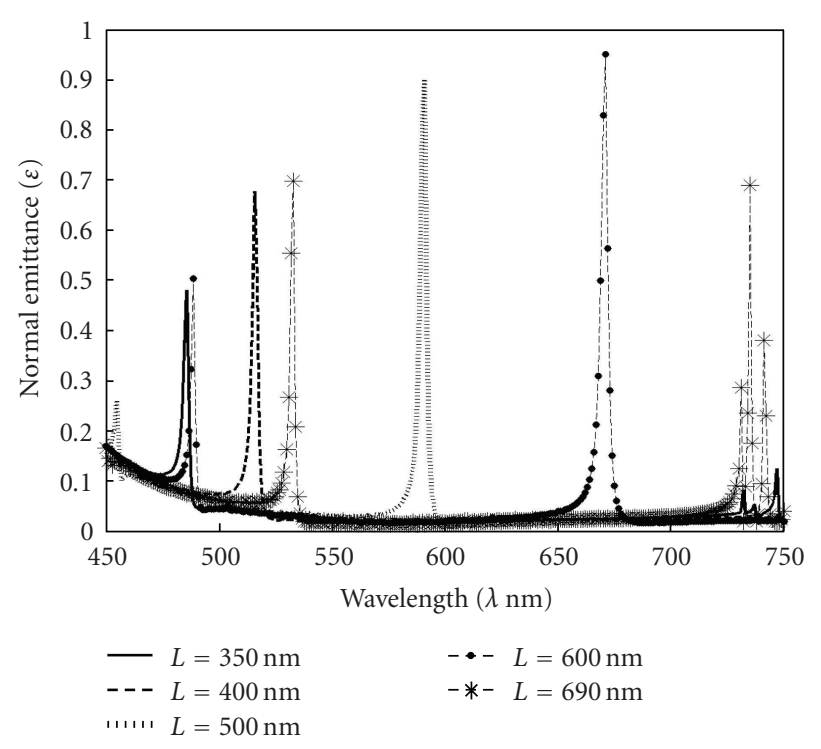

(a)

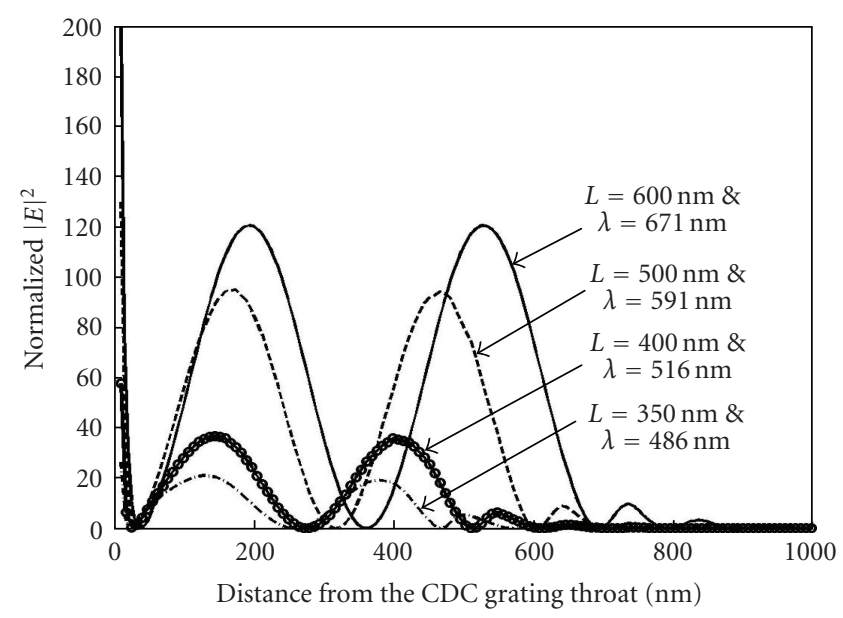

(b)

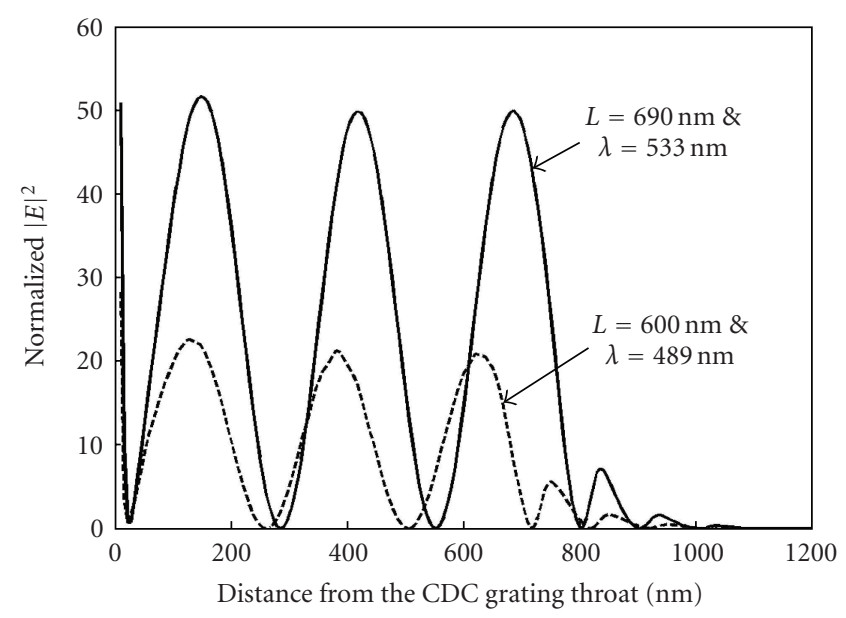

(c)

FIGURE 7: (a) Normal emittance spectrum with $p$-polarization in vacuum for Ag CDC grating having the parameters $\Lambda=250 \mathrm{~nm}, h=$ $100 \mathrm{~nm}, t=100 \mathrm{~nm}, g=5 \mathrm{~nm}$, and a photonic crystal having the parameters $a=100 \mathrm{~nm}, d_{1}=d_{2}=50$ nm with varying cavity. Electric field intensity distribution normalized to the incident along the line passing through the center of the CDC grating throat and into the photonic crystal with (b) $L=350 \mathrm{~nm}, L=400 \mathrm{~nm}, L=500 \mathrm{~nm}, L=600 \mathrm{~nm}$, and at respective higher normal emissive peak wavelengths (c) $L=600 \mathrm{~nm}$, $L=690 \mathrm{~nm}$, and at respective lower-normal emissive peak wavelengths.

is inversely proportional to the coherence time [27]. This would then suggest that the cavity length is a good degree of freedom for achieving a tunable monochromatic thermal emitter for any desired wavelength with large temporal coherence. Figure 6(b) shows the electric field intensity distribution normalized to the incident along a line passing through the center of the CDC grating throat and into the $1 \mathrm{D} \mathrm{PhC}$. The intensity variation in the cavity seems to be like a first harmonic standing wave between the Ag grating and 1D PhC. The magnitude of the electric field intensity variation in the cavity increases with the cavity length, suggesting that the increase in emissive peak magnitude is due to the increase in the cavity resonance strength. Also, in $1 \mathrm{D} \mathrm{PhC,}$ the electric field intensity decays within a smaller number of unit cells for larger cavity lengths. Hence, Figures 6(a)-6(b) show that the high absorption or emission is mainly due to the strong resonance in the cavity, and the condition for the resonant wavelength changes with the cavity length.

For a CDC, grating with $5 \mathrm{~nm}$ gap at the throat and $1 \mathrm{D}$ $\mathrm{PhC}$ with an increased cavity length from $320 \mathrm{~nm}$ to $350 \mathrm{~nm}$ has resulted in reducing the larger peaks present at higher wavelengths, as is evident in Figures 6(a) and 7(a). An additional increase in the cavity length to $400 \mathrm{~nm}$ has red shifted the spectral position of the emission peak present at lower 
wavelengths, and also the magnitude of the emissivity peak has increased. In addition, at this cavity length there are no emission peaks near the larger wavelengths. When the cavity length is increased to $500 \mathrm{~nm}$, then the emittance peak in the spectrum, as shown in Figure 7(a), has further red shifted and is almost in the middle of the wavelength range of interest. But, a closer look on the normal emission spectrum in the lower-wavelength range shows that there is a very small peak in the emission indicating that there could be an onset of even higher resonance in the cavity, as shown later. With the cavity length increased to $600 \mathrm{~nm}$, the larger emission peak in the spectrum has further red shifted, and the smaller emission peak has gained in strength with a similar red shift. But the normal emittance spectrum, for the cavity length of $690 \mathrm{~nm}$, has multiple emission peaks at higher wavelengths, and the emission peak at the lower wavelength has gained further in the magnitude with all of the emission peaks having red shift. Figure 7(b) shows the electric field intensity distribution normalized to the incident along the line passing through the center of the CDC throat and into the $1 \mathrm{D} \mathrm{PhC}$, at the spectral positions where the emission spectrum has large peaks for the cavity lengths of $350 \mathrm{~nm}, 400 \mathrm{~nm}, 500 \mathrm{~nm}$, and $600 \mathrm{~nm}$. From the figure, it can be seen that for all the cavity lengths considered, the resonance in the cavity is similar to a second harmonic resonance of a standing wave. Therefore, as the cavity length increases the spectral position of the emission peak due to the second harmonic resonance would have red shift, as observed similarly for the first harmonic resonance in Figures 6(a)-6(b). When the cavity lengths increase beyond a certain range, then there would be an onset of higher-order resonances at lower wavelengths and this is shown in Figure 7(c). In this figure, the normalized electric field intensity distribution is shown along the same line as the previous cases but for wavelengths where the spectral emission has peaks in the lower wavelength. The intensity distribution shows that the cavity has a resonance that is similar to a third harmonic standing wave.

In conclusion, we have shown that CDC metallic gratings with narrow and deep slits can have enhanced transmission resonances for wavelengths larger than the periodicity of the grating and have high-transmission resonances in a very narrow energy band. Also, it has been shown that a thermal emitter with a cavity surrounded by a CDC metallic grating and $1 \mathrm{D} \mathrm{PhC} \mathrm{has} \mathrm{very} \mathrm{sharp} \mathrm{spectral} \mathrm{emission} \mathrm{peak.} \mathrm{This} \mathrm{is}$ mainly due to the strong resonances in the cavity supported by the CDC grating and $1 \mathrm{D}$ PhC. Thus, the emitter is tunable to various wavelengths with the choice of right materials along with the parameters of the grating, cavity length, and 1D PhC. Hence, the metallic subwavelength gratings with CDC can have important applications in optical communications, biological sensing, and optoelectronics.

\section{ACKNOWLEDGMENTS}

Financial support from the US Air Force of Scientific Research (AFOSR) is greatly appreciated. This work was also supported in part by research grants from the US National Science Foundation. The authors appreciate the computer support from the Intel's Higher Education Program.

\section{REFERENCES}

[1] W. L. Barnes, T. W. Preist, S. C. Kitson, and J. R. Sambles, "Physical origin of photonic energy gaps in the propagation of surface plasmons on gratings," Physical Review B, vol. 54, no. 9, pp. 6227-6244, 1996.

[2] S. C. Kitson, W. L. Barnes, and J. R. Samblas, "Full photonic band gap for surface modes in the visible," Physical Review Letters, vol. 77, no. 13, pp. 2670-2673, 1996.

[3] H. A. Bethe, "Theory of diffraction by small holes," Physical Review, vol. 66, no. 7-8, pp. 163-182, 1944.

[4] T. W. Ebbesen, H. J. Lezec, H. F. Ghaemi, T. Thio, and P. A. Wolff, "Extraordinary optical transmission through subwavelength hole arrays," Nature, vol. 391, no. 6668, pp. 667$669,1998$.

[5] C. Genet and T. W. Ebbesen, "Light in tiny holes," Nature, vol. 445, no. 7123, pp. 39-46, 2007.

[6] F. J. García-Vidal and L. Martín-Moreno, "Transmission and focusing of light in one-dimensional periodically nanostructured metals," Physical Review B, vol. 66, no. 15, Article ID 155412, 10 pages, 2002.

[7] J. A. Dionne, L. A. Sweatlock, H. A. Atwater, and A. Polman, "Planar metal plasmon waveguides: frequency-dependent dispersion, propagation, localization, and loss beyond the free electron model," Physical Review B, vol. 72, no. 7, Article ID 075405, 11 pages, 2005.

[8] H. J. Lezec and T. Thio, "Diffracted evanescent wave model for enhanced and suppressed optical transmission through subwavelength hole arrays," Optics Express, vol. 12, no. 16, pp. 3629-3651, 2004.

[9] Q. Cao and P. Lalanne, "Negative role of surface plasmons in the transmission of metallic gratings with very narrow slits," Physical Review Letters, vol. 88, no. 5, Article ID 057403, 4 pages, 2002.

[10] W. L. Barnes, W. A. Murray, J. Dintinger, E. Devaux, and T. W. Ebbesen, "Surface plasmon polaritons and their role in the enhanced transmission of light through periodic arrays of subwavelength holes in a metal film," Physical Review Letters, vol. 92, no. 10, Article ID 107401, 4 pages, 2004.

[11] J. A. Porto, F. J. García-Vidal, and J. B. Pendry, "Transmission resonances on metallic gratings with very narrow slits," Physical Review Letters, vol. 83, no. 14, pp. 2845-2848, 1999.

[12] R. Carminati and J.-J. Greffet, "Near-field effects in spatial coherence of thermal sources," Physical Review Letters, vol. 82, no. 8, pp. 1660-1663, 1999.

[13] C. Henkel, K. Joulain, R. Carminati, and J.-J. Greffet, "Spatial coherence of thermal near fields," Optics Communications, vol. 186, no. 1-3, pp. 57-67, 2000.

[14] A. V. Shchegrov, K. Joulain, R. Carminati, and J.-J. Greffet, "Near-field spectral effects due to electromagnetic surface excitations," Physical Review Letters, vol. 85, no. 7, pp. 15481551, 2000.

[15] P. J. Hesketh, J. N. Zemel, and B. Gebhart, "Organ pipe radiant modes of periodic micromachined silicon surfaces," Nature, vol. 324, no. 6097, pp. 549-551, 1986.

[16] E. A. Vinogradov, G. N. Zhizhin, A. G. Mal'shukov, and V. I. Yudson, "Thermostimulated polariton emission of zinc selenide films on metal substrate," Solid State Communications, vol. 23, no. 12, pp. 915-921, 1977.

[17] M. Kreiter, J. Oster, R. Sambles, S. Herminghaus, S. MittlerNeher, and W. Knoll, "Thermally induced emission of light from a metallic diffraction grating, mediated by surface plasmons," Optics Communications, vol. 168, no. 1, pp. 117-122, 1999. 
[18] J.-J. Greffet, R. Carminati, K. Joulain, J.-P. Mulet, S. Mainguy, and Y. Chen, "Coherent emission of light by thermal sources," Nature, vol. 416, no. 6876, pp. 61-64, 2002.

[19] P. Ben-Abdallah, "Thermal antenna behavior for thin-film structures," Journal of the Optical Society of America A, vol. 21, no. 7, pp. 1368-1371, 2004.

[20] E. D. Palik, Ed., Handbook of Optical Constants of Solids, Academic Press, Orlando, Fla, USA, 1985.

[21] J.-J. Greffet and M. Nieto-Vesperinas, "Field theory for generalized bidirectional reflectivity: derivation of Helmholtz's reciprocity principle and Kirchhoff's law," Journal of the Optical Society of America A, vol. 15, no. 10, pp. 2735-2744, 1998.

[22] J. D. Jackson, Classical Electrodynamics, John Wiley \& Sons, New York, NY, USA, 3rd edition, 1999.

[23] D. R. Lide, Handbook of Chemistry and Physics, CRC Press, Cleveland, Ohio, USA, 58th edition, 1977.

[24] Y. Xie, A. R. Zakharian, J. V. Moloney, and M. Mansuripur, "Transmission of light through slit apertures in metallic films," Optics Express, vol. 12, no. 25, pp. 6106-6121, 2004.

[25] A. Lavrinenko, P. I. Borel, L. H. Frandsen, et al., "Comprehensive FDTD modelling of photonic crystal waveguide components," Optics Express, vol. 12, no. 2, pp. 234-248, 2004.

[26] X. Jiao, P. Wang, L. Tang, et al., "Fabry-Pérot-like phenomenon in the surface plasmons resonant transmission of metallic gratings with very narrow slits," Applied Physics B, vol. 80, no. 3, pp. 301-305, 2005.

[27] J. Le Gall, M. Olivier, and J.-J. Greffet, "Experimental and theoretical study of reflection and coherent thermal emission by a SiC grating supporting a surface-phonon polariton," Physical Review B, vol. 55, no. 15, pp. 10105-10114, 1997. 

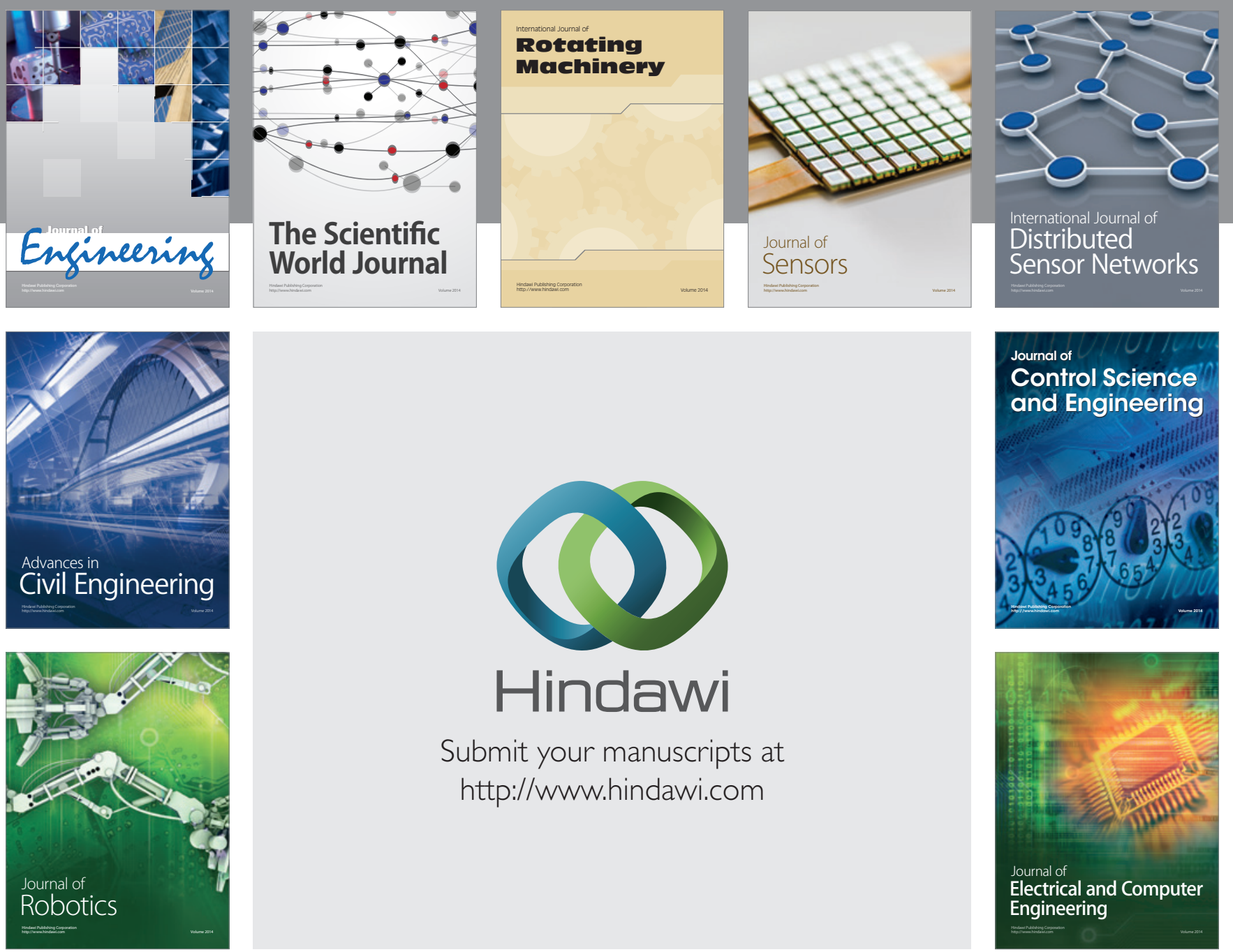

Submit your manuscripts at

http://www.hindawi.com
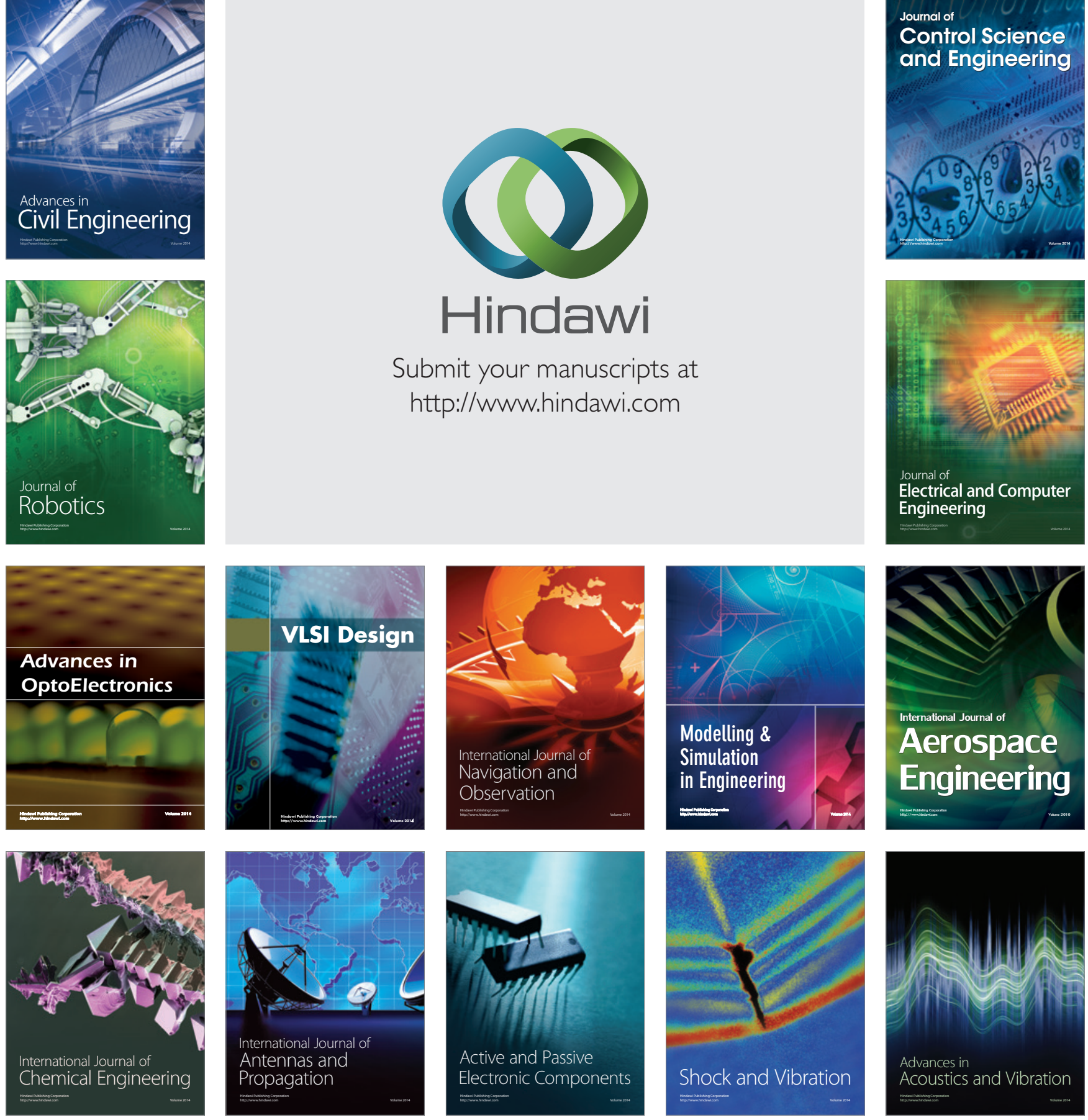